\title{
烷基杂环芳基二硫醚类化合物的合成及抗癌活性研究
}

\author{
龚景旭 盛丽姚励功李 佳周宇波郭跃伟* \\ (中国科学院上海药物研究所 新药研究国家重点实验室 上海 201203)
}

\begin{abstract}
摘要 通过 Bunte 盐与杂环芳基硫醇反应，得到了一系列的烷基芳基二硫醚类化合物. 得到的全部二硫醚类化合物对 人结肠癌细胞 HCT-116 抑制活性进行了体外评价, 发现所有化合物具有明显的体外抗肿瘤活性, 其半抑制浓度 $\mathrm{IC}_{50}$ 为 $4.8 \sim 17.7 \mu \mathrm{g} \cdot \mathrm{mL}^{-1}$.
\end{abstract}

关键词 二硫醚; 合成; 抗肿瘤活性

\section{Synthesis and Anti-tumor Activity Evaluation of Alkyl Heterocyclyl Disulfides}

\author{
Gong, Jingxu Sheng, Li Yao, Ligong Li, Jia Zhou, Yubo Guo, Yuewei* \\ (State Key Laboratory of Drug Research, Shanghai Institute of Materia Medica, Chinese Academy of Sciences, \\ Shanghai 201203)
}

\begin{abstract}
A series of alkyl heterocyclyl disulfides were prepared by reaction of the heterocyclyl thiol with Bunte salts. All the synthesized compounds showed interesting inhibit activities toward human colon carcinoma cells (HCT-116) in vitro. The $\mathrm{IC}_{50}$ values of the tested compounds ranged from $4.8 \mu \mathrm{g} \cdot \mathrm{mL}^{-1}$ to $17.7 \mu \mathrm{g} \bullet \mathrm{mL}^{-1}$.

Keywords disulfides; synthesis; anti-tumor activity
\end{abstract}

二硫醚类化合物显示出多方面良好的生物活性，如 抗菌 ${ }^{[1]}$ 、抗真菌 ${ }^{[2]}$ 、抗肿瘤活性 ${ }^{[3]}$ 、抗 HIV 活性 ${ }^{[4]}$ 等, 从 而引起广泛的关注. 其中二硫醚化合物吡硫醇 (Pyritinol)能促进脑内葡萄糖及氨基酸代谢, 改善全身 同化作用, 增强颈动脉血流量. 在临床上广泛用于脑震 荡综合症、脑外伤后遗症、脑炎及脑膜炎后遗症的头胀 疼、头晕、失眠、记忆力不集中、情绪变化等症 状的 改善, 也可用于脑动脉硬化、老年痴呆症等. 另一二硫 醚化合物 PX-12 是新型硫氧还原蛋白抑制剂, 硫氧还原 蛋白抑制剂是一类具有抗癌活性的药物, 目前 PX-12 即 将进入 II 期临床研究, 而 PX-12 是第一个进入临床的该 类药物 ${ }^{[5]}$.

近年来, 我们小组对红树林植物木榄(Bruguiera gymnorrhiza) 的化学成分进行了系统研究, 从中分离得 到多个二硫醚类化合物 ${ }^{[6,7]}$, 其中化合物木榄环六硫醇 (gymnorrhizol) 对 II 型糖尿病靶标蛋白酪氨酸酯酶 (PTP1B)具有明显的抑制活性，我们通过合成方法得到 了木榄环六硫醇极其类似物 ${ }^{[8,9]}$. 有关二硫醚类化合物 的良好生物活性引起了我们的研究兴趣，我们对此类化 合物进行了深入的研究, 并对其抗 PTP1B 活性、抗肿瘤 活性以及抗 HDAC 等活性进行了研究. 本文报道芳基 烷基不对称二硫醚类化合物的合成及其抗人结肠癌细 胞 HCT-116 活性研究. 合成路线如 Scheme 1 所示.

\section{1 实验部分}

\section{1 仪器和试剂}

MR 由 DRX-400 及 DP X-300 MHz 核磁共振仪测定, ${ }^{1} \mathrm{H} \mathrm{NMR}$ 位移值以気代溶剂中残存的 $\mathrm{CH}_{3} \mathrm{OH}(\delta 3.29)$ 及 $\mathrm{CHCl}_{3}\left(\delta\right.$ 7.26)为内标, 偶合常数 $J$ 的单位是 $\mathrm{Hz} ;{ }^{13} \mathrm{C}$

*E-mail: ywguo@mail.shcnc.ac.cn; Tel.: 021-50805813.

Received January 20, 2011; revised November 8, 2011; accepted November 28, 2011.

Project supported by the National S \& T Major Project (Nos. 2009ZX09301-001, 2009ZX09103-060), the Natural Science Foundation of China (Nos. 81072572, 21072204, 21021063), the National Marine "863" Project (No. 2011AA09070102), the Basic Research Project of Shanghai Science and Technology Commission (Nos. 09ZR1438000, 10540702900), and the Key Program of the Chinese Academy of Sciences (Nos. KSCX2-EW-R-15, KSCX2-YW-R-18).

国家新药创制重大专项(No. 2009ZX09301-00, 2009ZX09103-060)、国家自然科学基金(Nos. 81072572, 21072204, 21021063)、国家海洋 “863” 计划(No 2011AA09070102)、上海市科委基础研究(Nos. 09ZR1438000, 10540702900)和中国科学院重点(Nos. KSCX2-EW-R-15, KSCX2-YW-R-18)资助项目. 


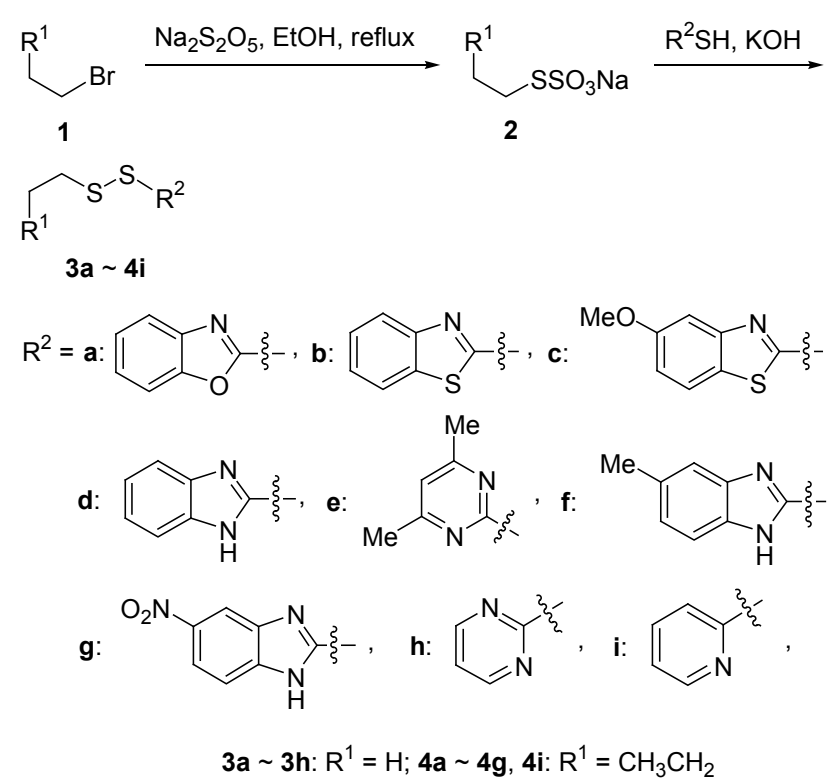

Scheme 1

NMR 位移值以気代溶剂中残存的 $\mathrm{CH}_{3} \mathrm{OH}(\delta$ 49.5)及 $\mathrm{CHCl}_{3}(\delta$ 77.0)为内标. E SIMS 和 HRESIMS 由 Q-TOF Micro LC-MS-MS 质谱仪测定. 试剂纯化参照文献[10]. 快速柱层析采用青岛海洋化工厂或烟台化工研究所实 验厂生产柱硅胶 H (100 200 目, 200 300 目, 10 40 $\left.\mu \mathrm{mol} \cdot \mathrm{L}^{-1}\right)$, 或者薄层层析硅胶 $\mathrm{H}$.

\subsection{Bunte 盐 2 的合成}

在反应瓶中加入 $10 \mathrm{mmol}$ 溴乙烷或正澳丁烷, 2.48 $\mathrm{g}$ 五水硫代硫酸钠, 用 $25 \mathrm{~mL} 75 \%$ 乙醇溶解, 加热回流, 取样分析, 硫代硫酸钠全部反应完后降至室温, 反应物 直接用于下一步反应.

\section{3 烷基杂环芳基二硫醚 3 和 4 的合成}

取 $10 \mathrm{mmol}$ 的硫醇与 $10 \mathrm{~mL}$ 水混合, 滴加 $30 \%$ $\mathrm{KOH}$ 至固体全部溶解后倒入前面的反应物中, 于室温 继续摚拌 $30 \mathrm{~min}$, 减压蒸去大部分的乙醇, 乙醚提取, 硫酸镁干燥, 过滤浓缩, 残余物经硅胶柱层析依次得到 化合物 3a $\sim \mathbf{4 i}$.

所得到的化合物 $3 \mathrm{a} \sim 3 \mathrm{z}, 4 \mathrm{c}$ 和 $4 \mathrm{e}$ 为新化合物, 其余 为已知化合物 ${ }^{[11 \sim 18]}$.

乙基(2-苯并噁唑)二硫醚(3a): 产率 75\%, 无色油状 物. ${ }^{1} \mathrm{H}$ NMR $\left(\mathrm{CDCl}_{3}, 300 \mathrm{MHz}\right) \delta: 1.43(\mathrm{t}, J=7.5 \mathrm{~Hz}, 3 \mathrm{H}$, $\left.\mathrm{CH}_{3}\right), 3.02$ (q, $\left.J=7.5 \mathrm{~Hz}, 2 \mathrm{H}, \mathrm{CH}_{2}\right), 7.26 \sim 7.32(\mathrm{~m}, 2 \mathrm{H}$, $\mathrm{ArH}), 7.48 \sim 7.51(\mathrm{~m}, 1 \mathrm{H}, \mathrm{ArH}), 7.66 \sim 7.69(\mathrm{~m}, 1 \mathrm{H}, \mathrm{ArH})$;

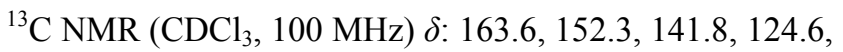
124.5, 119.3, 1110.2, 33.2, 13.9; ESIMS $m / z$ (\%): 211.9 $\left([\mathrm{M}+1]^{+}, 100\right)$; HRESIMS calcd for $\mathrm{C}_{9} \mathrm{H}_{9} \mathrm{NOS}_{2} \mathrm{Na}$ 234.0023 , found 234.0001 . 乙基(2-苯并噻唑)二硫醚(3b): 产率 72\%，无色油状 物. ${ }^{1} \mathrm{H}$ NMR $\left(\mathrm{CDCl}_{3}, 300 \mathrm{MHz}\right) \delta: 1.41(\mathrm{t}, J=7.4 \mathrm{~Hz}, 3 \mathrm{H}$, $\mathrm{CH}_{3}$ ), 2.98 (q, $\left.J=7.4 \mathrm{~Hz}, 2 \mathrm{H}, \mathrm{CH}_{2}\right), 7.30 \sim 7.35(\mathrm{~m}, 2 \mathrm{H}$, ArH), $7.40 \sim 7.45$ (m, 1H, ArH), 7.79 (dd, $J=7.8,0.9 \mathrm{~Hz}$, $1 \mathrm{H}, \mathrm{ArH}), 7.86$ (dd, $J=7.8,0.6 \mathrm{~Hz}, 1 \mathrm{H}, \mathrm{ArH}) ;$ ESI-MS $m / z$ (\%): $227.9\left([\mathrm{M}+1]^{+}, 100\right)$.

乙基(5-甲氧基-2-苯并噻唑)二硫醚(3c)：产率 72\%, 无色油状物. ${ }^{1} \mathrm{H}$ NMR $\left(\mathrm{CDCl}_{3}, 300 \mathrm{MHz}\right) \delta: 1.41(\mathrm{t}, J=$ $7.2 \mathrm{~Hz}, 3 \mathrm{H}, \mathrm{CH}_{3}$ ), 2.97 (q, $J=7.2 \mathrm{~Hz}, 2 \mathrm{H}, \mathrm{CH}_{2}$ ), 3.86 (s, $\left.3 \mathrm{H}, \mathrm{CH}_{3} \mathrm{O}\right), 6.96(\mathrm{dd}, J=8.7,2.4 \mathrm{~Hz}, 1 \mathrm{H}, \mathrm{ArH}), 7.36(\mathrm{~d}$, $J=2.7 \mathrm{~Hz}, 1 \mathrm{H}, \mathrm{ArH}), 7.63(\mathrm{~d}, J=8.7 \mathrm{~Hz} 1 \mathrm{H}, \operatorname{ArH}) ;{ }^{13} \mathrm{C}$ NMR $\left(\mathrm{CDCl}_{3}, 100 \mathrm{MHz}\right) \delta: 174.0,158.8,156.2,127.6$, $121.2,114.3,104.8,55.5,33.3,29.6,14.2$; ESIMS $\mathrm{m} / \mathrm{z}$ (\%): $257.9\left([\mathrm{M}+1]^{+}, 100\right)$; HRESIMS calcd for $\mathrm{C}_{10} \mathrm{H}_{11^{-}}$ $\mathrm{NOS}_{3} \mathrm{Na} 279.9900$, found 279.9922 .

乙基(2-苯并咪唑)二硫醚(3d): 产率 68\%，白色固 体. ${ }^{1} \mathrm{H}$ NMR $\left(\mathrm{CD}_{3} \mathrm{OD}, 300 \mathrm{MHz}\right) \delta: 1.33(\mathrm{t}, J=7.2 \mathrm{~Hz}$, $\left.3 \mathrm{H}, \mathrm{CH}_{3}\right), 2.93\left(\mathrm{q}, J=7.2 \mathrm{~Hz}, 2 \mathrm{H}, \mathrm{CH}_{2}\right), 7.20 \sim 7.23(\mathrm{~m}$, $2 \mathrm{H}, \mathrm{ArH}), 7.49 \sim 7.52(\mathrm{~m}, 2 \mathrm{H}, \mathrm{ArH}) ;{ }^{13} \mathrm{C}$ NMR $\left(\mathrm{CDCl}_{3}\right.$, $100 \mathrm{MHz}) \delta: 174.0,158.8,156.2,127.6,121.2,114.3$, $104.8,55.5,33.3,29.6,14.2,151.5,123.9,115.5,34.4$, 14.3; ESIMS $m / z(\%): 210.9\left([\mathrm{M}+1]^{+}, 100\right)$; HRESIMS calcd for $\mathrm{C}_{9} \mathrm{H}_{10} \mathrm{~N}_{2} \mathrm{~S}_{2} \mathrm{Na} 233.0183$, found 233.0165 .

乙基(4,6-二甲基-2-嘧啶)二硫醚(3e): 产率 74\%, 无 色油状物. ${ }^{1} \mathrm{H}$ NMR $\left(\mathrm{CDCl}_{3}, 300 \mathrm{MHz}\right) \delta: 1.34$ (t, $J=7.4$ $\left.\mathrm{Hz}, 3 \mathrm{H}, \mathrm{CH}_{3}\right), 2.44$ (s, 6H, $\mathrm{CH}_{3}$ ), 2.89 (q, $J=7.4 \mathrm{~Hz}, 2 \mathrm{H}$, $\left.\mathrm{CH}_{2}\right), 7.26$ (s, $\left.1 \mathrm{H}, \mathrm{ArH}\right) ;{ }^{13} \mathrm{C}$ NMR $\left(\mathrm{CD}_{3} \mathrm{OD}, 100 \mathrm{MHz}\right) \delta$ : 170.5, 167.6, 117.0, 32.2, 23.8, 13.9; ESIMS $\mathrm{m} / \mathrm{z}$ (\%): $200.9\left([\mathrm{M}+1]^{+}, 100\right)$; HRESIMS calcd for $\mathrm{C}_{8} \mathrm{H}_{12} \mathrm{~N}_{2} \mathrm{~S}_{2} \mathrm{Na}$ 223.0340, found 223.0356

乙基(5-甲基-2-苯并咪唑)二硫醚(3f)：产率 70\%，无 色油状物. ${ }^{1} \mathrm{H}$ NMR $\left(\mathrm{CD}_{3} \mathrm{OD}, 300 \mathrm{MHz}\right) \delta: 1.33(\mathrm{t}, J=6.9$ $\left.\mathrm{Hz}, 3 \mathrm{H}, \mathrm{CH}_{3}\right), 2.34$ (s, 3H, $\left.\mathrm{CH}_{3}\right), 2.90$ (q, $J=6.9 \mathrm{~Hz}, 2 \mathrm{H}$, $\left.\mathrm{CH}_{2}\right), 6.98 \sim 7.10(\mathrm{~m}, 4 \mathrm{H}, \mathrm{ArH}) ;{ }^{13} \mathrm{C}$ NMR $\left(\mathrm{CD}_{3} \mathrm{OD}, 100\right.$ MHz) $\delta$ : 134.2, 125.0, 34.4, 21.4, 14.3; ESIMS $m / z(\%)$ : $224.9\left([\mathrm{M}+1]^{+}, 100\right)$; HRESIMS calcd for $\mathrm{C}_{10} \mathrm{H}_{13} \mathrm{~N}_{2} \mathrm{~S}_{2}$ 225.0520 , found 225.0535.

乙基(5-硝基-2-苯并咪唑)二硫醚(3g)：产率 $65 \%$, 黄色固体. ${ }^{1} \mathrm{H}$ NMR $\left(\mathrm{CDCl}_{3}, 300 \mathrm{MHz}\right) \delta$ : 1.40 (t, $J=7.2$ $\left.\mathrm{Hz}, 3 \mathrm{H}, \mathrm{CH}_{3}\right), 2.95$ (q, $\left.J=7.2 \mathrm{~Hz}, 2 \mathrm{H}, \mathrm{CH}_{2}\right), 7.60$ (d, $J=$ $7.8 \mathrm{~Hz}, 1 \mathrm{H}, \mathrm{ArH}), 8.19$ (dd, $J=8.7,1.8 \mathrm{~Hz}, 1 \mathrm{H}, \mathrm{ArH}), 8.48$ (s, 1H, ArH); ESI-MS $m / z(\%): 256.0\left([\mathrm{M}+1]^{+}, 100\right)$.

乙基(2-嘧啶)二硫醚(3h): 产率 69\%, 无色油状物. ${ }^{1} \mathrm{H}$ NMR $\left(\mathrm{CDCl}_{3}, 300 \mathrm{MHz}\right) \delta: 1.34$ (t, $J=7.4 \mathrm{~Hz}, 3 \mathrm{H}$, $\left.\mathrm{CH}_{3}\right), 2.89$ (q, $\left.J=7.4 \mathrm{~Hz}, 2 \mathrm{H}, \mathrm{CH}_{2}\right), 7.05 \sim 7.09(\mathrm{~m}, 1 \mathrm{H}$, 
ArH), $8.60 \sim 8.62(\mathrm{~m}, 2 \mathrm{H}, \mathrm{ArH}) ;$ ESI-MS $m / z(\%): 172.9$ $\left([\mathrm{M}+1]^{+}, 100\right)$.

正丁基(2-苯并噁唑)二硫醚(4a): 产率 73\%, 无色油 状物. ${ }^{1} \mathrm{H}$ NMR $\left(\mathrm{CDCl}_{3}, 300 \mathrm{MHz}\right) \delta: 0.92(\mathrm{t}, J=7.2 \mathrm{~Hz}$, $\left.3 \mathrm{H}, \mathrm{CH}_{3}\right), 1.42 \sim 1.49\left(\mathrm{~m}, 2 \mathrm{H}, \mathrm{CH}_{2}\right), 1.70 \sim 1.80(\mathrm{~m}, 2 \mathrm{H}$, $\left.\mathrm{CH}_{2}\right), 3.00\left(\mathrm{t}, J=7.4 \mathrm{~Hz}, 2 \mathrm{H}, \mathrm{CH}_{2}\right), 7.29 \sim 7.32(\mathrm{~m}, 2 \mathrm{H}$, ArH), $7.48 \sim 7.50(\mathrm{~m}, 1 \mathrm{H}, \mathrm{ArH}), 7.66 \sim 7.69(\mathrm{~m}, 1 \mathrm{H}, \mathrm{ArH})$; ESI-MS $m / z$ (\%): 239.9 ([M+1] $\left.]^{+}, 100\right)$.

正丁基(2-苯并噻唑)二硫醚(4b): 产率 63\%, 无色油 状物. ${ }^{1} \mathrm{H}$ NMR $\left(\mathrm{CDCl}_{3}, 300 \mathrm{MHz}\right) \delta: 0.93(\mathrm{t}, J=7.6 \mathrm{~Hz}$, $\left.3 \mathrm{H}, \mathrm{CH}_{3}\right), 1.39 \sim 1.49\left(\mathrm{~m}, 2 \mathrm{H}, \mathrm{CH}_{2}\right), 1.69 \sim 1.80(\mathrm{~m}, 2 \mathrm{H}$, $\left.\mathrm{CH}_{2}\right), 2.97\left(\mathrm{t}, J=7.4 \mathrm{~Hz}, 2 \mathrm{H}, \mathrm{CH}_{2}\right), 7.30 \sim 7.35(\mathrm{~m}, 1 \mathrm{H}$, ArH), $7.40 \sim 7.46(\mathrm{~m}, 1 \mathrm{H}, \mathrm{ArH}), 7.79 \sim 7.88(\mathrm{~m}, 2 \mathrm{H}, \mathrm{ArH})$; ESI-MS $m / z(\%): 256.0\left([\mathrm{M}+1]^{+}, 100\right)$

正丁基(5-甲氧基-2-苯并噻唑)二硫醚(4c)：产率 $76 \%$, 无色油状物. ${ }^{1} \mathrm{H} \mathrm{NMR}\left(\mathrm{CDCl}_{3}, 300 \mathrm{MHz}\right) \delta: 0.92$ (t, $\left.J=7.5 \mathrm{~Hz}, 3 \mathrm{H}, \mathrm{CH}_{3}\right), 1.39 \sim 1.49\left(\mathrm{~m}, 2 \mathrm{H}, \mathrm{CH}_{2}\right), 1.69 \sim$ $1.80\left(\mathrm{~m}, 2 \mathrm{H}, \mathrm{CH}_{2}\right), 2.96\left(\mathrm{t}, J=7.4 \mathrm{~Hz}, 2 \mathrm{H}, \mathrm{CH}_{2}\right), 3.86(\mathrm{~s}$, $\left.3 \mathrm{H}, \mathrm{CH}_{3} \mathrm{O}\right), 6.97(\mathrm{dd}, J=8.7,2.4 \mathrm{~Hz}, 1 \mathrm{H}, \mathrm{ArH}), 7.36(\mathrm{~d}$, $J=2.4 \mathrm{~Hz}, 1 \mathrm{H}, \mathrm{ArH}), 7.65(\mathrm{~d}, J=8.7 \mathrm{~Hz}, 1 \mathrm{H}, \operatorname{ArH}) ;{ }^{13} \mathrm{C}$ NMR $\left(\mathrm{CDCl}_{3}, 100 \mathrm{MHz}\right) \delta: 174.2,158.9,156.2,127.6$, $121.3,114.3,104.8,55.5,39.2,30.9,21.5$, 13.5; ESI-MS $\mathrm{m} / \mathrm{z}$ (\%): $286.0\left([\mathrm{M}+1]^{+}, 100\right)$; HRESIMS calcd for $\mathrm{C}_{12} \mathrm{H}_{16} \mathrm{NOS}_{3} 286.0394$, found 286.0374 .

正丁基(2-苯并咪唑)二硫醚(4d): 产率 74\%, 白色固 体. ${ }^{1} \mathrm{H}$ NMR $\left(\mathrm{CD}_{3} \mathrm{OD}, 300 \mathrm{MHz}\right) \delta: 0.86$ (t, $J=7.4 \mathrm{~Hz}$, $\left.3 \mathrm{H}, \mathrm{CH}_{3}\right), 1.29 \sim 1.43\left(\mathrm{~m}, 2 \mathrm{H}, \mathrm{CH}_{2}\right), 1.62 \sim 1.72(\mathrm{~m}, 2 \mathrm{H}$, $\mathrm{CH}_{2}$ ), 2.89 (t, $\left.J=7.4 \mathrm{~Hz}, 2 \mathrm{H}, \mathrm{CH}_{2}\right), 7.22 \sim 7.25(\mathrm{~m}, 2 \mathrm{H}$, ArH), $7.50 \sim 7.58$ (m, 2H, ArH); ESI-MS $m / z$ (\%): 239.0 $\left([\mathrm{M}+1]^{+}, 100\right)$.

正丁基(4,6-二甲基-2-嘧啶)二硫梄(4e): 产率 70\%, 无色油状物. ${ }^{1} \mathrm{H}$ NMR $\left(\mathrm{CDCl}_{3}, 300 \mathrm{MHz}\right) \delta: 0.89$ (t, $J=$ $\left.7.2 \mathrm{~Hz}, 3 \mathrm{H}, \mathrm{CH}_{3}\right), 1.41 \sim 1.48\left(\mathrm{~m}, 2 \mathrm{H}, \mathrm{CH}_{2}\right), 1.65 \sim 1.70$ (m, 2H, $\mathrm{CH}_{2}$ ), 2.44 (s, 6H, $\mathrm{CH}_{3}$ ), 2.87 (t, $J=7.2 \mathrm{~Hz}, 2 \mathrm{H}$, $\left.\mathrm{CH}_{2}\right), 6.77$ (s, $\left.1 \mathrm{H}, \mathrm{ArH}\right) ;{ }^{13} \mathrm{C} \mathrm{NMR}\left(\mathrm{CDCl}_{3}, 100 \mathrm{MHz}\right) \delta$ : 170.5, 167.6, 116.9, 38.3, 30.6, 23.8, 21.5, 13.6; ESI-MS $m / z$ (\%): $229.0\left([\mathrm{M}+1]^{+}, 100\right)$; HRESIMS calcd for $\mathrm{C}_{10} \mathrm{H}_{17} \mathrm{~N}_{2} \mathrm{~S}_{2} 229.0833$ found 229.0819 .

正丁基(5-甲基-2-苯并咪唑)二硫醚(4f): 产率 66\%, 无色油状物. ${ }^{1} \mathrm{H}$ NMR $\left(\mathrm{CDCl}_{3}, 300 \mathrm{MHz}\right) \delta: 0.86$ (t, $J=$ $\left.7.4 \mathrm{~Hz}, 3 \mathrm{H}, \mathrm{CH}_{3}\right), 1.33 \sim 1.41\left(\mathrm{~m}, 2 \mathrm{H}, \mathrm{CH}_{2}\right), 1.62 \sim 1.69$ (m, $2 \mathrm{H}, \mathrm{CH}_{2}$ ), 2.45 (s, 3H, $\mathrm{CH}_{3}$ ), 2.84 (t, $J=7.5 \mathrm{~Hz}, 2 \mathrm{H}$, $\left.\mathrm{CH}_{2}\right), 7.05(\mathrm{~d}, J=8.1 \mathrm{~Hz}, 1 \mathrm{H}, \mathrm{ArH}), 7.32(\mathrm{~s}, 1 \mathrm{H}, \mathrm{ArH})$, $7.44(\mathrm{~d}, J=8.1 \mathrm{~Hz}, 1 \mathrm{H}, \mathrm{ArH})$; ESI-MS $m / z$ (\%): 253.0 $\left([\mathrm{M}+1]^{+}, 100\right)$. 正丁基(5-硝基-2-苯并咪唑)二硫醚(4g)：产率 64\%, 黄色固体. ${ }^{1} \mathrm{H}$ NMR $\left(\mathrm{CDCl}_{3}, 300 \mathrm{MHz}\right) \delta: 0.88(\mathrm{t}, J=7.2$ $\left.\mathrm{Hz}, 3 \mathrm{H}, \mathrm{CH}_{3}\right), 1.36 \sim 1.44\left(\mathrm{~m}, 2 \mathrm{H}, \mathrm{CH}_{2}\right), 1.64 \sim 1.72(\mathrm{~m}$, $2 \mathrm{H}, \mathrm{CH}_{2}$ ), 2.90 (t, $\left.J=7.5 \mathrm{~Hz}, 2 \mathrm{H}, \mathrm{CH}_{2}\right), 7.60$ (d, $J=7.8$ $\mathrm{Hz}, 1 \mathrm{H}, \mathrm{ArH}), 8.18$ (d, J=7.8 Hz, 1H, ArH), 8.47 (s, $1 \mathrm{H}$, $\mathrm{ArH})$; ESI-MS $m / z$ (\%): $284.0\left([\mathrm{M}+1]^{+}, 100\right)$.

正丁基(2-吡啶)二硫醚(4i): 产率 77\%, 无色油状物, ${ }^{1} \mathrm{H}$ NMR $\left(\mathrm{CDCl}_{3}, 300 \mathrm{MHz}\right) \delta: 0.91(\mathrm{t}, J=7.2 \mathrm{~Hz}, 3 \mathrm{H}$, $\left.\mathrm{CH}_{3}\right), 1.37 \sim 1.44\left(\mathrm{~m}, 2 \mathrm{H}, \mathrm{CH}_{2}\right), 1.62 \sim 1.69\left(\mathrm{~m}, 2 \mathrm{H}, \mathrm{CH}_{2}\right)$, $2.79\left(\mathrm{t}, J=7.2 \mathrm{~Hz}, 2 \mathrm{H}, \mathrm{CH}_{2}\right), 7.05 \sim 7.09(\mathrm{~m}, 1 \mathrm{H}, \mathrm{ArH})$, $7.61 \sim 7.66(\mathrm{~m}, 1 \mathrm{H}, \operatorname{ArH}), 7.73(\mathrm{dd}, J=8.4,1.2 \mathrm{~Hz}, 1 \mathrm{H}$, ArH), $8.44 \sim 8.46(\mathrm{~m}, 1 \mathrm{H}, \mathrm{ArH}) ; \mathrm{ESI}-\mathrm{MS} m / z(\%): 199.9$ $\left([\mathrm{M}+1]^{+}, 100\right)$.

\section{4 体外抗肿瘤活性实验}

运用 MTT 法对所合成的化合物进行了体外抗肿瘤 活性实验. 实验用的肿瘤细胞为人结肠癌细胞 (HCT-116).

\section{2 结果与讨论}

\section{1 合成方法讨论}

不对称二硫醚化合物常用的方法有硫醇与烷氧羰 基硫基氯反应、硫醇与 Bunte 盐反应 ${ }^{[19]}$ 、硫醇与 1-氯苯 并三唑 ${ }^{[20]} 反$ 应等, 二硫醚交换 ${ }^{[21]}$ 等. Sirakawa 等 ${ }^{[22]}$ 报道 了以烷基硫醇和硫艮为起始物, 经过氧化氢氧化生成烷 基硫代异硫腿盐酸盐，再与杂环芳基硫醇反应，生成二 硫醚化合物, Kirkpatrick 等 ${ }^{[16]}$ 对此方法进行了改进, 该 方法要使用有恶臭气味的硫醇化合物, 实验过程中对环 境和人体都会造成伤害. 我们对此类化合物的合成方法 进行了改进, 采用卤代物与硫代硫酸钠反应, 生成的 Bunte 盐再与杂环芳基硫醇反应, 得到了目标化合物, 避免了具有恶臭的乙硫醇或丁硫醇的使用，该方法是一 种即环保又安全的方法.

\section{2 体外抗肿瘤活性研究结果}

体外抗肿瘤活性测试结果(表 1)表明: 所有化合物 都显示一定的抗肿瘤活性, 其 $\mathrm{IC}_{50}$ 达到 $10 \mu \mathrm{g} \cdot \mathrm{mL}^{-1}$ 左 右. 根据测试结果, 我们进行了初步的构效关系研究, 结果表明 $\mathbf{3}$ 和 4 系列化合物抗肿瘤活性没有显著的差别, 表明烷基链长对于活性没有显著的影响. 不同的杂环对 于活性有不同的影响, 其中以吡啶取代的 $4 \mathbf{i}$ 活性最弱, 苯并噁坐取代的 3a, $4 a$ 和苯并噻唑取代的 $3 b, 4 b$ 次之. 而苯并咪唑、嘧啶等具有 1,3 -二氮杂结构化合物的活性 相对要强，而在其苯环上含有甲基、硝基等取代基后， 化合物的活性又有所增强. 到目前为止，有关二硫醚类 化合物的抗肿瘤活性的报道还不是很多, 通过我们的研 
究, 初步确立了此类化合物的抗肿瘤活性构效关系. 在 此基础上，我们将进一步优化结构，并测试更多的肿瘤 模型，以寻找具有更高活性、更低毒副作用的二硫醚类 抗肿瘤药物.

表 13 和 4 系列化合物的体外抗肿瘤活性测试结果 $\left[\mathrm{IC}_{50} /\right.$ $\left.\left(\mu \mathrm{g} \cdot \mathrm{mL}^{-1}\right)\right]$

Table 1 In vitro inhibition activities of tumor for compounds 3 and $4\left[\mathrm{IC}_{50} /\left(\mu \mathrm{g} \cdot \mathrm{mL}^{-1}\right)\right]$

\begin{tabular}{ccccccccc}
\hline Compd. & 3a & 3b & 3c & 3d & 3e & 3f & 3g & 3h \\
\hline HCT-116 & 10.4 & 10.8 & 7.3 & 11.1 & 10.5 & 6.3 & 4.8 & 11.1 \\
\hline \hline Compd. & 4a & $\mathbf{4 b}$ & $\mathbf{4 c}$ & $\mathbf{4 d}$ & $\mathbf{4 e}$ & $\mathbf{4 f}$ & $\mathbf{4 g}$ & $\mathbf{4 i}$ \\
\hline HCT-116 & 14.0 & 13.7 & 10.1 & 5.40 & 9.2 & 7.3 & 6.8 & 17.7 \\
\hline
\end{tabular}

\section{References}

[1] Park, H. B.; Kwon, H. C.; Lee, C.-H.; Yang, H. O. J. Nat. Prod. 2009, 72, 248

[2] Turos, E.; Revell, K. D.; Ramaraju, P.; Gergeres, D. A.; Greenhalgh, K.; Young, A.; Sathyanarayan, N.; Dickey, S.; Lim, D.; Alhamadsheh, M. M.; Reynolds, K. Bioorg. Med. Chem. 2008, 16, 6501.

[3] (a) Hunter, R.; Kaschula, C. H.; Parker, I. M.; Caira, M. R.; Richards, P.; Travis, S.; Taute, F.; Qwebani, T. Bioorg. Med. Chem. Lett. 2008, 18, 5277.

(b) O'Donnell, G.; Poeschl, R.; Zimhony, O.; Gunaratnam, M.; Moreira, J. B. C.; Neidle, S.; Evangelopoulos, D.; Bhakta, S.; Malkinson, J. P.; Boshoff, H. I.; Lenaerts, A.; Gibbons, S. J. Nat. Prod. 2009, 72, 360

(c) Ryu, E. K.; Choe, Y. S.; Byun, S. S.; Lee, K.-H.; Chi, D. Y.; Choi, Y.; Kim, B.-T. Bioorg. Med. Chem. 2004, 12, 859.
[4] Cesarini, S.; Spallarossa, A.; Ranise, A.; Schenone, S.; Bruno, O.; P Colla, L.; Casula, L.; Collu, G.; Sanna, G.; Loddo, R. Bioorg. Med. Chem. 2008, 16, 6353.

[5] Cragg, G. M.; Grothaus, P. G.; Newman, D. J. Chem. Rev. 2009, 109, 3012.

[6] Sun, Y.-Q.; Guo, Y.-W. Tetrahedron Lett. 2004, 45, 5533.

[7] Liu H.-L.; Shen, X.; Jiang, H.; Guo, Y.-W. Chin. J. Org. Chem. 2008, 28, 246 (in Chinese). (刘海利, 沈旭, 蒋华良, 郭跃伟, 有机化学, 2008, 28, 246.)

[8] Gong, J.-X.; Shen, X.; Yao, L.-G.; Jiang, H.-L.; Krohn, K.; Guo, Y.-W. Org. Lett. 2007, 9, 1715.

[9] Gong, J.-X.; Sun, Y.-Q.; Wang, J.-D.; Guo, Y.-W. Chin. J. Org. Chem. 2008, 28, 246 (in Chinese). (龚景旭, 孙燕秋, 王继栋, 郭跃伟, 有机化学, 2008, 28, 252.)

[10] Perrin, D. D.; Armarego, W.L. F.; Perrin, D. R. Purification of Laboratory Chemicals, Pergamon Press, Oxford, 1980.

[11] Zhang, J.; Yang, S.; Liu, W.; Xue, Q. Tribol. Lett. 1999, 7, 173.

[12] Tanaka, K.; Ajiki, K. Tetrahedron Lett. 2004, 45, 5677.

[13] Ozelli, R. N.; Scheer, H. DE 2456900, 1976 [Chem. Abstr. 1976, $85,125527]$.

[14] Xue, Q.; Zhang, J.; Liu, W.; Yang, S. Tribol. Lett. 1999, 7, 27.

[15] Kirkpatrick, D. L. US 6552060, 2003 [Chem. Abstr. 2003, 138, 314629].

[16] Kikrpatrick, D. L.; Jimale, M. L.; King, K. M.; Chen, T. Eur. J. Med. Chem. 1992, 27, 33.

[17] Jayasuriya, N.; Regen, S. Tetrahedron Lett. 1992, 33, 451.

[18] Stellenboom, N.; Hunter, R.; Caira, M. R. Tetrahedron 2010, 66, 3228.

[19] Hiver, P.; Dicko, A.; Paquer, D. Tetrahedron Lett. 1994, 35, 9569.

[20] Hunter, R.; Caira, M.; Stellenboom, N. J. Org. Chem. 2006, 71, 8268.

[21] Tanaka, K.; Ajiki, K. Tetrahedron Lett. 2004, 45, 5677.

[22] Sirakawa, K.; Aki, O.; Tsujikawa, T.; Tsuda, T. Chem. Pharm. Bull. 1970, 18, 235. 